\title{
Iterative Approximation of the Minimal and Maximal Positive Solutions for Multipoint Fractional Boundary Value Problem on an Unbounded Domain
}

\author{
Guotao Wang, ${ }^{1}$ Sanyang Liu, ${ }^{1}$ and Lihong Zhang ${ }^{2}$ \\ ${ }^{1}$ Department of Applied Mathematics, Xidian University, Xian, Shaanxi 710071, China \\ ${ }^{2}$ School of Mathematics and Computer Science, Shanxi Normal University, Linfen, Shanxi 041004, China \\ Correspondence should be addressed to Sanyang Liu; liusanyang@126.com
}

Received 27 May 2013; Revised 11 November 2013; Accepted 18 November 2013; Published 28 January 2014

Academic Editor: Aurelian Gheondea

Copyright (C) 2014 Guotao Wang et al. This is an open access article distributed under the Creative Commons Attribution License, which permits unrestricted use, distribution, and reproduction in any medium, provided the original work is properly cited.

By employing the monotone iterative method, this paper not only establishes the existence of the minimal and maximal positive solutions for multipoint fractional boundary value problem on an unbounded domain, but also develops two computable explicit monotone iterative sequences for approximating the two positive solutions. An example is given for the illustration of the main result.

\section{Introduction}

The fractional calculus has been recognized as an effective modeling methodology for describing hereditary properties of various materials and processes widely. For a lot of applications, we refer the reader to the books [1-5]. For some new development on the topic, see [6-17] and the references therein.

Recently, there has been a significant development on boundary value problems for fractional differential equations on infinite intervals; see papers [18-26], in which authors are devoted to investigating the existence of solutions and positive solutions by employing some fixed point theorems, Leray-Schauder nonlinear alternative theorem, or fixed point index theory.

By using Schauder's fixed point theorem combined with the diagonalization method, Arara et al. [18] studied the existence of the bounded solution of the following problem on infinite intervals:

$$
\begin{gathered}
{ }^{C} D_{0+}^{\alpha} y(t)=f(t, y(t)), \quad 1<\alpha \leq 2, \\
y(0)=y_{0}, \quad y \text { is bounded on } J,
\end{gathered}
$$

where $t \in J=[0,+\infty), f \in C(J \times \mathbb{R}, \mathbb{R}), y_{0} \in \mathbb{R}$, and ${ }^{C} D_{0+}^{\alpha}$ is the Caputo fractional derivative of order $\alpha$.
In [19], Zhao and Ge investigated the existence of positive solutions for the following fractional boundary value problem by employing the Leray-Schauder nonlinear alternative theorem:

$$
\begin{aligned}
& D_{0+}^{\alpha} u(t)+f(t, u(t))=0, \quad 1<\alpha \leq 2, \\
& u(0)=0, \quad \lim _{t \rightarrow+\infty} D_{0+}^{\alpha-1}=\beta u(\xi),
\end{aligned}
$$

where $t \in J=[0,+\infty), f \in C(J \times \mathbb{R},[0,+\infty)), 0 \leq \xi$, $\eta<\infty$, and $D_{0+}^{\alpha}$ is the standard Riemann-Liouville fractional derivative.

Liang and Zhang [20] were concerned with the following nonlinear fractional differential equations with multipoint fractional boundary conditions on an unbounded domain:

$$
\begin{gathered}
D^{\alpha} u(t)+a(t) f(t, u(t))=0, \quad 0<t<\infty, \\
u(0)=u^{\prime}(0)=0, \\
D^{\alpha-1} u(+\infty)=\sum_{i=1}^{m-2} \beta_{i} u\left(\xi_{i}\right),
\end{gathered}
$$

where $J=[0,+\infty), 2<\alpha \leq 3, D^{\alpha}$ denotes the Riemann-Liouville fractional derivative, $0<\xi_{1}<\xi_{2}<\cdots<$ $\xi_{m-2}<+\infty$, and $\beta_{i}>0, i=1,2, \ldots, m-2$, satisfy 
$0<\sum_{i=1}^{m-2} \beta_{i} \xi_{i}^{\alpha-1}<\Gamma(\alpha)$. By using the fixed point index theory, authors gave sufficient conditions for the existence of multiple positive solutions to the above multi-point fractional boundary value problem.

However, very interesting and important question is "If we know the existence of the solution, how can we find it?" This question motivates us to reconsider problem (3). In this paper, we not only establish the existence of two positive solutions for problem (3), but also develop two computable explicit monotone iterative sequences for approximating the minimal and maximal positive solutions of (3), which is indeed an important and useful contribution to the existing literature on the topic. In addition, to start our work, we employ the monotone iterative method, which is different from the ones used in [18-26]. Let us state that this method was widely used for nonlinear problem; see, for instance, [2738].

\section{Preliminaries and Several Lemmas}

In this section, we present some useful definitions and related theorems.

Definition 1 (see [2]). The Riemann-Liouville fractional derivative of order $\delta$ for a continuous function $f$ is defined by

$$
\begin{aligned}
D^{\delta} f(t)= & \frac{1}{\Gamma(n-\delta)}\left(\frac{d}{d t}\right)^{n} \\
& \times \int_{0}^{t}(t-s)^{n-\delta-1} f(s) d s, \quad n=[\delta]+1,
\end{aligned}
$$

provided the right-hand side is pointwise defined on $(0, \infty)$ and $[\delta]$ is the integer part of $\delta$.

Definition 2 (see [2]). The Riemann-Liouville fractional integral of order $\delta$ for a function $f$ is defined as

$$
I^{\delta} f(t)=\frac{1}{\Gamma(\delta)} \int_{0}^{t}(t-s)^{\delta-1} f(s) d s, \quad \delta>0,
$$

provided that such integral exists.

Lemma 3 (see $[20])$. Let $h \in C([0,+\infty))$. For $2<\alpha<3$, the fractional boundary value problem

$$
\begin{gathered}
D^{\alpha} u(t)+h(t)=0, \\
u(0)=u^{\prime}(0)=0, \\
D^{\alpha-1} u(+\infty)=\sum_{i=1}^{m-2} \beta_{i} u\left(\xi_{i}\right)
\end{gathered}
$$

has a unique solution

$$
u(t)=\int_{0}^{+\infty} G(t, s) h(s) d s,
$$

where

$$
G(t, s)=G^{*}(t, s)+G^{* *}(t, s),
$$

with

$$
\begin{gathered}
G^{*}(t, s)=\frac{1}{\Gamma(\alpha)} \begin{cases}t^{\alpha-1}-(t-s)^{\alpha-1}, & 0 \leq s \leq t<+\infty \\
t^{\alpha-1}, & 0 \leq t \leq s<+\infty\end{cases} \\
G^{* *}(t, s)=\frac{\sum_{i=1}^{m-2} \beta_{i} t^{\alpha-1}}{\Gamma(\alpha)-\sum_{i=1}^{m-2} \beta_{i} \xi_{i}^{\alpha-1}} G^{*}\left(\xi_{i}, s\right) .
\end{gathered}
$$

Lemma 4 (see $[20])$. For $(s, t) \in[0,+\infty) \times[0,+\infty)$, then Green's function $G(t, s)$ has the following properties:

(1)

$$
0 \leq G(t, s) \leq L t^{\alpha-1}
$$

(2)

$$
\frac{G(t, s)}{1+t^{\alpha-1}} \leq L
$$

where

$$
L=\frac{1}{\Gamma(\alpha)}+\frac{\sum_{i=1}^{m-2} \beta_{i} \xi_{m-2}^{\alpha-1}}{\Gamma(\alpha)\left(\Gamma(\alpha)-\sum_{i=1}^{m-2} \beta_{i} \xi_{i}^{\alpha-1}\right)} .
$$

For the forthcoming analysis, we will use a Banach space:

$$
X=\left\{u \in C(J, \mathbb{R}): \sup _{t \in J} \frac{|u(t)|}{1+t^{\alpha-1}}<+\infty\right\},
$$

equipped with the norm

$$
\|u\|_{X}=\sup _{t \in J} \frac{|u(t)|}{1+t^{\alpha-1}} .
$$

Define a cone $P \subset X$ by

$$
P=\{u \in X: u(t) \geq 0, t \in J\}
$$

and an operator $Q: X \rightarrow X$ as follows:

$$
Q u(t)=\int_{0}^{\infty} G(t, s) a(s) f(s, u(s)) d s .
$$

Observe that multi-point fractional boundary value problem (3) has a solution if and only if the integral operator $Q$ has a fixed point.

\section{Main Results}

In this section, we shall construct two explicit monotone iterative sequences which converge to the minimal and maximal positive solutions of (3).

Theorem 5. Assume that the following conditions hold:
$\left(\mathrm{H}_{1}\right) f \in C(J \times J, J), f(t, 0)$ \# 0 on any subinterval of $J$, and when $u$ is bounded, $f\left(t,\left(1+t^{\alpha-1}\right) u\right)$ is bounded on $J$;

$\left(\mathrm{H}_{2}\right) a: J \rightarrow J$ does not identically vanish on any subinterval of $J$ and $0<\int_{0}^{+\infty} a(t) d t<\infty$; 
$\left(\mathrm{H}_{3}\right) f(t, \cdot)$ is nondecreasing for any $t \in J$, and there exists a constant $b>0$, such that $f\left(t,\left(1+t^{\alpha-1}\right) u\right) \leq$ $b / L \int_{0}^{\infty} a(t) d t$ for $(t, u) \in J \times[0, b]$.

Then the multi-point fractional boundary value problem (3) has the minimal and maximal positive solutions $v^{*}, u^{*}$ in $\left(0, b t^{\alpha-1}\right]$, which can be obtained by the following two explicit monotone iterative sequences:

$$
\begin{array}{r}
v_{n+1}=\int_{0}^{+\infty} G(t, s) a(s) f\left(s, v_{n}(s)\right) d s \\
\quad \text { with initial value } v_{0}(t)=0, \\
u_{n+1}=\int_{0}^{+\infty} G(t, s) a(s) f\left(s, u_{n}(s)\right) d s \\
\text { with initial value } u_{0}(t)=b t^{\alpha-1} .
\end{array}
$$

Moreover,

$$
\begin{aligned}
v_{0} & \leq v_{1} \leq \cdots \leq v_{n} \cdots \leq v^{*} \leq \cdots \\
& \leq u^{*} \cdots \leq u_{n} \leq \cdots \leq u_{1} \leq u_{0} .
\end{aligned}
$$

Proof. By a similar process used in [20], it is easy to show that $Q: P \rightarrow P$ is completely continuous.

Now denote $B=\left\{u \in P,\|u\|_{X} \leq b\right\}$; then we have $Q(B) \subset$ $B$. In fact, let $u \in B$; then by $\left(\mathrm{H}_{3}\right)$ and (12), we have

$$
\begin{aligned}
\|Q u\|_{X} & =\sup _{t \in J} \int_{0}^{+\infty} \frac{G(t, s)}{1+t^{\alpha-1}}|a(s) f(s, u(s))| d s \\
& \leq L \int_{0}^{+\infty} a(s) d s \cdot \frac{b}{L \int_{0}^{+\infty} a(s) d s}=b .
\end{aligned}
$$

That is, $Q(B) \subset B$.

Denote that $v_{0}(t)=0, v_{1}=Q 0=Q v_{0}$, and $v_{2}=Q^{2} 0=$ $Q v_{1}$, for all $t \in J$. Since $v_{0}(t)=0 \in B$ and $Q: B \rightarrow B$, then $v_{1} \in Q(B) \subset B$ and $v_{2} \in Q(B) \subset B$. So, we have

$$
v_{1}(t)=(Q 0)(t) \geq 0=v_{0}(t), \quad \forall t \in J .
$$

By condition $\left(\mathrm{H}_{3}\right)$, for $u, v \in B$ and $u \geq v$, we have

$$
\begin{aligned}
Q u(t) & =\int_{0}^{\infty} G(t, s) a(s) f(s, u(s)) d s \\
& \geq \int_{0}^{\infty} G(t, s) a(s) f(s, v(s)) d s=Q v(t) .
\end{aligned}
$$

This proves that $Q$ is a nondecreasing operator.

So, we have

$$
v_{2}(t)=\left(Q v_{1}\right)(t) \geq\left(Q v_{0}\right)(t)=v_{1}(t), \quad \forall t \in J
$$

By the induction, define $v_{n+1}=Q v_{n}, n=0,1,2, \ldots$ Then the sequence $\left\{v_{n}\right\}_{n=1}^{\infty} \subset Q(B) \subset B$ and satisfies the following relation:

$$
v_{n+1}(t) \geq v_{n}(t), \quad \forall t \in J, \quad n=0,1,2, \ldots
$$

In view of the complete continuity of the operator $Q$ and $v_{n+1}=Q v_{n}$, then $\left\{v_{n}\right\}_{n=1}^{\infty}$ is relative compact. That is, $\left\{v_{n}\right\}_{n=1}^{\infty}$ has a convergent subsequence $\left\{v_{n_{k}}\right\}_{k=1}^{\infty}$ and there exists a $v^{*} \in$ $B$ such that $v_{n_{k}} \rightarrow v^{*}$ as $k \rightarrow \infty$. This, together with (23), holds $\lim _{n \rightarrow \infty} v_{n}=v^{*}$.

Since $Q$ is continuous and $v_{n+1}=Q v_{n}$, then we have $\mathrm{Q} v^{*}=v^{*}$. That is, $v^{*}$ is a fixed point of the operator $Q$.

Denote that $u_{0}(t)=b t^{\alpha-1}, u_{1}=Q u_{0}$, and $u_{2}=Q^{2} u_{0}=$ $Q u_{1}$, for all $t \in J$. Since $u_{0}(t) \in B$ and $Q: B \rightarrow B$, then $u_{1} \in Q(B) \subset B$ and $u_{2} \in Q(B) \subset B$. By $\left(\mathrm{H}_{3}\right)$, we have

$$
\begin{aligned}
u_{1}(t) & =\int_{0}^{+\infty} G(t, s) a(s) f\left(s, u_{0}(s)\right) d s \\
& \leq \int_{0}^{+\infty} L t^{\alpha-1} a(s) d s \cdot \frac{b}{\int_{0}^{+\infty} L a(s) d s} \\
& =b t^{\alpha-1}=u_{0}(t), \quad \forall t \in J .
\end{aligned}
$$

Since $Q$ is nondecreasing, then we have

$$
u_{2}(t)=\left(Q u_{1}\right)(t) \leq\left(Q u_{0}\right)(t)=u_{1}(t), \quad \forall t \in J
$$

By the induction, define $u_{n+1}=Q u_{n}, n=0,1,2, \ldots$ Then the sequence $\left\{u_{n}\right\}_{n=1}^{\infty} \subset Q(B) \subset B$ and satisfies the following relation:

$$
u_{n+1}(t) \leq u_{n}(t), \quad \forall t \in J, n=0,1,2, \ldots
$$

With an analysis exactly parallel to the proving process of $\lim _{n \rightarrow \infty} v_{n}=v^{*}$, we have that there exists a $u^{*} \in B$ such that $\lim _{n \rightarrow \infty} u_{n}=u^{*}$.

Since $Q$ is continuous and $u_{n+1}=Q u_{n}$, we have $Q u^{*}=u^{*}$. That is, $u^{*}$ is a fixed point of the operator $Q$.

Now, we are in a position to show that $u^{*}$ and $v^{*}$ are the maximal and minimal positive solutions of (3) in $\left(0, b t^{\alpha-1}\right]$.

Let $w \in\left[0, b t^{\alpha-1}\right]$ be any solution of (3). That is $Q w=$ $w$. Noting that $Q$ is nondecreasing and $v_{0}(t)=0 \leq w(t) \leq$ $b t^{\alpha-1}=u_{0}(t)$, then we have $v_{1}(t)=Q v_{0}(t) \leq w(t) \leq Q u_{0}(t)=$ $u_{1}(t)$, for all $t \in J$.

Similarly, we can obtain

$$
v_{n}(t) \leq w(t) \leq u_{n}(t), \quad \forall t \in J, n=0,1,2, \ldots
$$

Since $u^{*}=\lim _{n \rightarrow \infty} u_{n}$ and $v^{*}=\lim _{n \rightarrow \infty} v_{n}$, it follows from (23) (27) that

$$
\begin{aligned}
v_{0} & \leq v_{1} \leq \cdots \leq v_{n} \cdots \leq v^{*} \\
& \leq w \leq u^{*} \cdots \leq u_{n} \leq \cdots \leq u_{1} \leq u_{0} .
\end{aligned}
$$

Since $f(t, 0) \quad \not \equiv 0$, for all $t \in J$, then 0 is not a solution of problem (3). Thus, by (28), we know that $u^{*}$ and $v^{*}$ are the maximal and minimal positive solutions of (3) in $\left(0, b t^{\alpha-1}\right]$, which can be obtained by the corresponding iterative sequences in (17).

This completes the proof. 


\section{Example}

Example 1. Take $\alpha=5 / 2, \beta_{1}=3 / 10, \beta_{2}=1 / 5, \xi_{1}=1 / 4$, and $\xi_{2}=1$. Consider the following boundary value problem:

$$
\begin{gathered}
D^{5 / 2} u(t)+e^{-t} f(t, u(t))=0, \quad t \in(0,+\infty) \\
u(0)=u^{\prime}(0)=0, \\
D^{3 / 2} u(+\infty)=\frac{3}{10} u\left(\frac{1}{4}\right)+\frac{1}{5} u(1)
\end{gathered}
$$

where $a(t)=e^{-t}$ and

$$
f(t, u)= \begin{cases}\frac{1}{100\left(1+t^{4}\right)}+\frac{1}{10}\left(\frac{u}{1+t^{3 / 2}}\right)^{5}, & 0 \leq u \leq 1, \\ \frac{1}{100\left(1+t^{4}\right)}+\frac{1}{10}\left(\frac{1}{1+t^{3 / 2}}\right)^{5}, & u>1 .\end{cases}
$$

Now, we show that $f\left(t,\left(1+t^{\alpha-1}\right) u\right)$ is bounded on $J$ when $u$ is bounded. Since

$$
\begin{aligned}
& f\left(t,\left(1+t^{3 / 2}\right) u\right) \\
& \quad= \begin{cases}\frac{1}{100\left(1+t^{4}\right)}+\frac{1}{10} u^{5}, & 0 \leq u \leq 1, \\
\frac{1}{100\left(1+t^{4}\right)}+\frac{1}{10}\left(\frac{1}{1+t^{3 / 2}}\right)^{5}, & u>1 .\end{cases}
\end{aligned}
$$

Then we have $f\left(t,\left(1+t^{3 / 2}\right) u\right) \leq 11 / 100$. So condition $\left(\mathrm{H}_{1}\right)$ holds. holds.

In view of $\int_{0}^{+\infty} a(t) d t=\int_{0}^{+\infty} e^{-t} d t=1$, condition $\left(\mathrm{H}_{2}\right)$

By a simple computation, we have that $\Gamma(\alpha)=\Gamma(5 / 2)=$ $3 \sqrt{\pi} / 4$ and $L=(1 / \Gamma(\alpha))+\left(\sum_{i=1}^{m-2} \beta_{i} \xi_{m-2}^{\alpha-1} / \Gamma(\alpha)(\Gamma(\alpha)-\right.$ $\left.\left.\sum_{i=1}^{m-2} \beta_{i} \xi_{i}^{\alpha-1}\right)\right) \approx 1.096741$. Taking $b=1$, it follows that

$$
\begin{aligned}
& f\left(t,\left(1+t^{3 / 2}\right) u\right) \leq 0.11<\frac{1}{1.0968} \\
& \leq \frac{b}{L \int_{0}^{\infty} a(s) d s}, \\
& \text { for }(t, u) \in J \times[0,1] .
\end{aligned}
$$

Hence, condition $\left(\mathrm{H}_{3}\right)$ holds. Thus all conditions of Theorem 5 are satisfied. Therefore, the fractional boundary value problem (29) has the minimal and maximal positive solutions in $\left(0, t^{3 / 2}\right]$, which can be obtained by two explicit monotone iterative sequences.

\section{Conflict of Interests}

The authors declare that there is no conflict of interests regarding the publication of this paper.

\section{Acknowledgments}

The authors would like to express their gratitude to the anonymous reviewers and editors for their valuable comments and suggestions which improved the quality of the original paper. This work is supported by the NNSF of China (no. 61373174) and the Natural Science Foundation for Young Scientists of Shanxi Province, China (no. 2012021002-3).

\section{References}

[1] I. Podlubny, Fractional Differential Equations, vol. 198 of Mathematics in Science and Engineering, Academic Press, San Diego, Calf, USA, 1999.

[2] A. A. Kilbas, H. M. Srivastava, and J. J. Trujillo, Theory and Applications of Fractional Differential Equations, vol. 204 of North-Holland Mathematics Studies, Elsevier Science B.V., Amsterdam, The Netherlands, 2006.

[3] V. Lakshmikantham, S. Leela, and J. V. Devi, Theory of Fractional Dynamic Systems, Cambridge Scientific, Cambridge, UK, 2009.

[4] J. Sabatier, O. P. Agrawal, and J. A. T. Machado, Eds., Advances in Fractional Calculus: Theoretical Developments and Applications in Physics and Engineering, Springer, Dordrecht, The Netherlands, 2007.

[5] D. Baleanu, K. Diethelm, E. Scalas, and J. J. Trujillo, Fractional Calculus Models and Numerical Methods, vol. 3 of Series on Complexity, Nonlinearity and Chaos, World Scientific, Hackensack, NJ, USA, 2012.

[6] F. Jarad, T. Abdeljawad, and D. Baleanu, "Stability of $q$-fractional non-autonomous systems," Nonlinear Analysis: Real World Applications, vol. 14, no. 1, pp. 780-784, 2013.

[7] G.-C. Wu and D. Baleanu, "Variational iteration method for the Burgers' flow with fractional derivatives-new Lagrange multipliers," Applied Mathematical Modelling, vol. 37, no. 9, pp. 6183-6190, 2013.

[8] E. Hernández, D. O’Regan, and K. Balachandran, “On recent developments in the theory of abstract differential equations with fractional derivatives," Nonlinear Analysis: Theory, Methods \& Applications, vol. 73, no. 10, pp. 3462-3471, 2010.

[9] A. Cabada and G. Wang, "Positive solutions of nonlinear fractional differential equations with integral boundary value conditions," Journal of Mathematical Analysis and Applications, vol. 389, no. 1, pp. 403-411, 2012.

[10] G. Wang, A. Cabada, and L. Zhang, "Integral boundary value problem for nonlinear differential equations of fractional order on an unbounded domain," to appear in Journal of Integral Equations and Applications.

[11] G. Wang, B. Ahmad, and L. Zhang, "Impulsive anti-periodic boundary value problem for nonlinear differential equations of fractional order," Nonlinear Analysis: Theory, Methods \& Applications, vol. 74, no. 3, pp. 792-804, 2011.

[12] Z. Bai and W. Sun, "Existence and multiplicity of positive solutions for singular fractional boundary value problems," Computers \& Mathematics with Applications, vol. 63, no. 9, pp. 1369-1381, 2012.

[13] J. Dabas and A. Chauhan, "Existence and uniqueness of mild solution for an impulsive neutral fractional integro-differential equation with infinite delay," Mathematical and Computer Modelling, vol. 57, no. 3-4, pp. 754-763, 2013.

[14] R. Sakthivel, N. I. Mahmudov, and J. J. Nieto, "Controllability for a class of fractional-order neutral evolution control systems," Applied Mathematics and Computation, vol. 218, no. 20, pp. 10334-10340, 2012.

[15] B. Ahmad, J. J. Nieto, A. Alsaedi, and M. El-Shahed, "A study of nonlinear Langevin equation involving two fractional orders in 
different intervals," Nonlinear Analysis: Real World Applications, vol. 13, no. 2, pp. 599-606, 2012.

[16] B. Ahmad and S. K. Ntouyas, "Existence results for nonlocal boundary value problems of fractional differential equations and inclusions with strip conditions," Boundary Value Problems, vol. 2012, article 55, 21 pages, 2012.

[17] R. P. Agarwal, D. O’Regan, and S. Staněk, "Positive solutions for mixed problems of singular fractional differential equations," Mathematische Nachrichten, vol. 285, no. 1, pp. 27-41, 2012.

[18] A. Arara, M. Benchohra, N. Hamidi, and J. J. Nieto, "Fractional order differential equations on an unbounded domain," Nonlinear Analysis: Theory, Methods \& Applications, vol. 72, no. 2, pp. 580-586, 2010.

[19] X. Zhao and W. Ge, "Unbounded solutions for a fractional boundary value problems on the infinite interval," Acta Applicandae Mathematicae, vol. 109, no. 2, pp. 495-505, 2010.

[20] S. Liang and J. Zhang, "Existence of multiple positive solutions for $m$-point fractional boundary value problems on an infinite interval," Mathematical and Computer Modelling, vol. 54, no. 56, pp. 1334-1346, 2011.

[21] S. Liang and J. Zhang, "Existence of three positive solutions of $m$-point boundary value problems for some nonlinear fractional differential equations on an infinite interval," Computers \& Mathematics with Applications, vol. 61, no. 11, pp. 3343-3354, 2011.

[22] X. Su, "Solutions to boundary value problem of fractional order on unbounded domains in a Banach space," Nonlinear Analysis: Theory, Methods \& Applications, vol. 74, no. 8, pp. 2844-2852, 2011.

[23] X. Su and S. Zhang, "Unbounded solutions to a boundary value problem of fractional order on the half-line," Computers \& Mathematics with Applications, vol. 61, no. 4, pp. 1079-1087, 2011.

[24] R. P. Agarwal, M. Benchohra, S. Hamani, and S. Pinelas, "Boundary value problems for differential equations involving Riemann-Liouville fractional derivative on the half-line," Dynamics of Continuous, Discrete \& Impulsive Systems. Series A, vol. 18, no. 2, pp. 235-244, 2011.

[25] L. Zhang, B. Ahmad, G. Wang, R. P. Agarwal, M. Al-Yami, and W. Shammakh, "Nonlocal integrodifferential boundary value problem for nonlinear fractional differential equations on an unbounded domain," Abstract and Applied Analysis, vol. 2013, Article ID 813903, 5 pages, 2013.

[26] G. Wang, B. Ahmad, and L. Zhang, "A coupled system of nonlinear fractional differential equations with multipoint fractional boundary conditions on an unbounded domain," Abstract and Applied Analysis, vol. 2012, Article ID 248709, 11 pages, 2012.

[27] G. S. Ladde, V. Lakshmikantham, and A. S. Vatsala, Monotone Iterative Techniques for Nonlinear Differential Equations, Monographs, Advanced Texts and Surveys in Pure and Applied Mathematics, 27, Pitman, Boston, Mass, USA, 1985.

[28] J. J. Nieto, "An abstract monotone iterative technique," Nonlinear Analysis: Theory, Methods \& Applications, vol. 28, no. 12, pp. 1923-1933, 1997.

[29] J. D. Ramírez and A. S. Vatsala, "Monotone iterative technique for fractional differential equations with periodic boundary conditions," Opuscula Mathematica, vol. 29, no. 3, pp. 289-304, 2009.

[30] G. Wang, "Monotone iterative technique for boundary value problems of a nonlinear fractional differential equation with deviating arguments," Journal of Computational and Applied Mathematics, vol. 236, no. 9, pp. 2425-2430, 2012.
[31] G. Wang, R. P. Agarwal, and A. Cabada, "Existence results and the monotone iterative technique for systems of nonlinear fractional differential equations," Applied Mathematics Letters, vol. 25, no. 6, pp. 1019-1024, 2012.

[32] G. Wang, D. Baleanu, and L. Zhang, "Monotone iterative method for a class of nonlinear fractional differential equations," Fractional Calculus and Applied Analysis, vol. 15, no. 2, pp. 244-252, 2012.

[33] L. Zhang, B. Ahmad, G. Wang, and R. P. Agarwal, "Nonlinear fractional integro-differential equations on unbounded domains in a Banach space," Journal of Computational and Applied Mathematics, vol. 249, pp. 51-56, 2013.

[34] T. Jankowski, "Fractional equations of Volterra type involving a Riemann-Liouville derivative," Applied Mathematics Letters, vol. 26, no. 3, pp. 344-350, 2013.

[35] F. A. McRae, "Monotone iterative technique and existence results for fractional differential equations," Nonlinear Analysis: Theory, Methods \& Applications, vol. 71, no. 12, pp. 6093-6096, 2009.

[36] Z. Wei, Q. Li, and J. Che, "Initial value problems for fractional differential equations involving Riemann-Liouville sequential fractional derivative," Journal of Mathematical Analysis and Applications, vol. 367, no. 1, pp. 260-272, 2010.

[37] Z. Liu, J. Sun, and I. Szántó, "Monotone iterative technique for Riemann-Liouville fractional integro-differential equations with advanced arguments," Results in Mathematics, vol. 63, no. 3-4, pp. 1277-1287, 2013.

[38] X. Zhang, L. Liu, Y. Wu, and Y. Lu, “The iterative solutions of nonlinear fractional differential equations," Applied Mathematics and Computation, vol. 219, no. 9, pp. 4680-4691, 2013. 


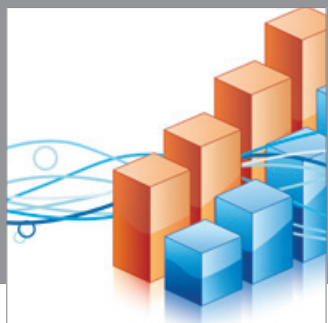

Advances in

Operations Research

mansans

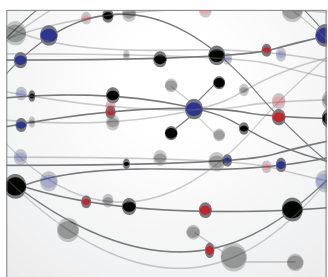

The Scientific World Journal
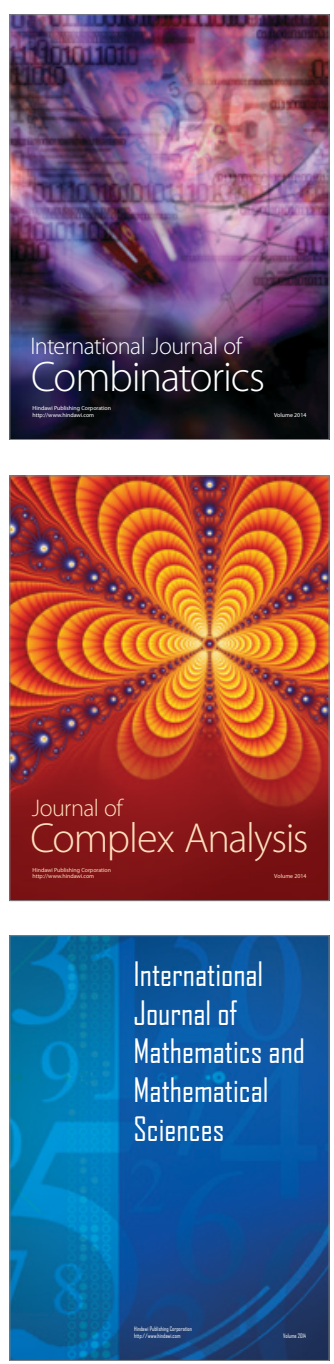
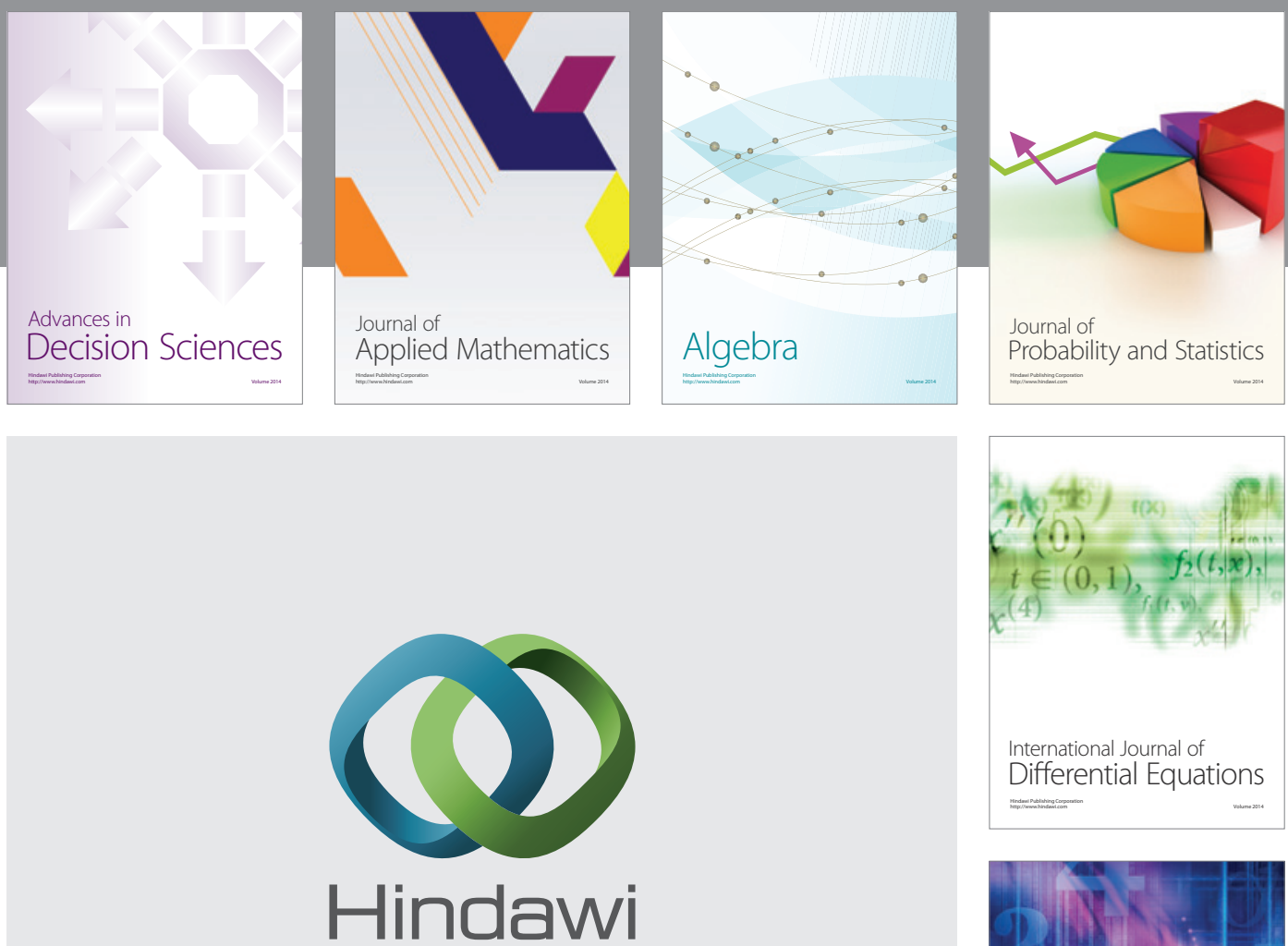

Submit your manuscripts at http://www.hindawi.com
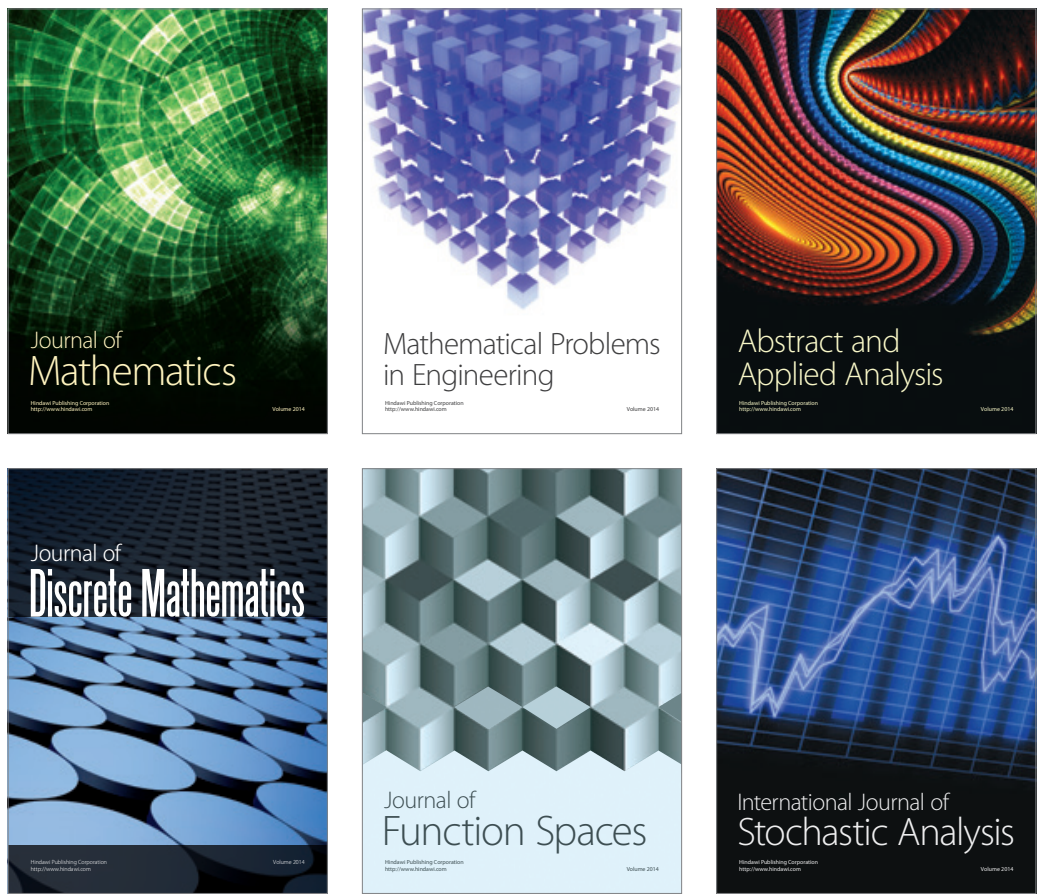

Journal of

Function Spaces

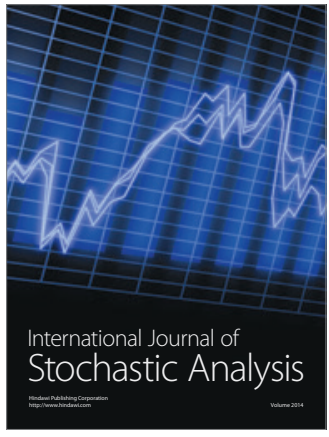

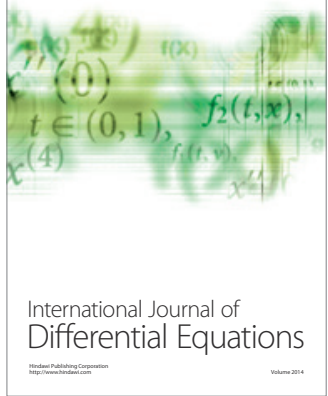
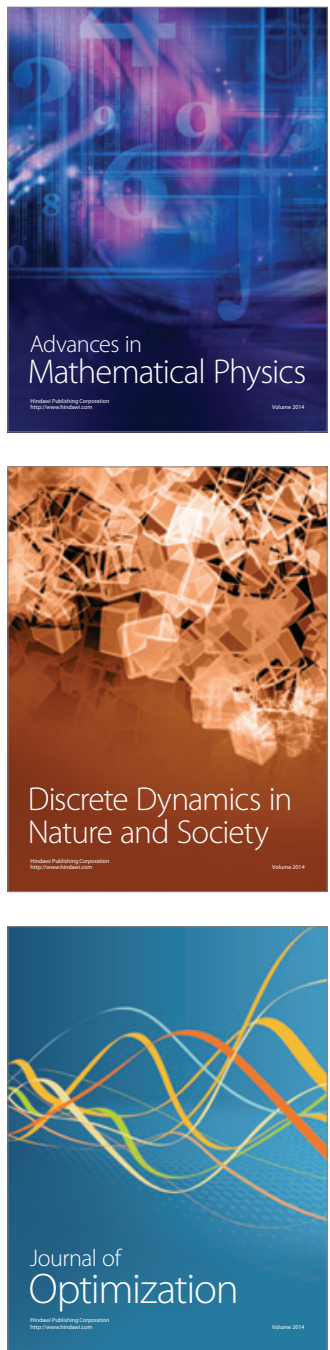